\title{
Initiation of Sporulation in Saccharomyces cerevisiae. Mutations Preventing Initiation
}

\author{
By GEOFFREY R. CALVERT AND IAN W. DAWES* \\ Department of Microbiology, University of Edinburgh, Edinburgh EH9 3JG, UK
}

(Received 24 November 1983)

Mutants of Saccharomyces cerevisiae that are unable to initiate sporulation, but can continue vegetative growth under conditions in which the wild-type strain sporulates, have been isolated and characterized. The mutations arose spontaneously as suppressors of the spdl mutations, restoring the ability of spdl mutants to grow on glycerol, and also spontaneously in cultures of a wild-type diploid strain undergoing sporulation in continuous culture. The mutations all conferred asporogeny, and were recessive in this respect to the wild-type, but dominant in acting as suppressors of the spdl mutation. They fell into three complementation groups which corresponded to three unlinked loci, designated spo50, spo51 and spo53. None of these mutations was closely linked to the other initiation mutations defined by the $s p d 1, s p d 3, s p d 4, c d c 25, c d c 28$ loci, nor to the cell size control mutations whil and whi2. Loose linkage was detected between spdl and spo53, and spo50, spd 3 and spo53 were linked to their respective centromeres. The spo50, spo51 and spo53 mutations are not nonsense suppressors. Mutations in all three genes conferred similar highly pleiotropic phenotypes including: asporogeny; dominant suppression of both spdl and spd3 mutations; aberrant cell morphology and viability loss on starvation; constitutive ability to reduce tetrazolium (which is subject to carbon source repression in the wild-type); and complete repression of the synthesis of several polypeptides that are subject to carbon source repression in the wild-type strain and derepressed in spdl mutants derepressed for sporulation. A diploid strain homozygous for the spo50 mutation did not undergo either premeiotic DNA replication or meiotic recombination when transferred to sporulation media.

\section{INTRODUCTION}

To study the mechanisms controlling initiation of yeast sporulation, it is important to obtain as many different classes of mutation as possible which affect the process. Mutations leading to derepression of sporulation have been described previously (Dawes, 1975; Shilo et al., 1978; Vezhinet et al., 1979; Dawes \& Calvert, 1984). Initiation mutants that fail to sporulate under conditions in which the wild-type does are intrinsically more difficult to recognize. A large number of asporogenous mutants have been isolated (Esposito \& Esposito, 1969) and partially characterized, but from the way these mutants were selected it is not possible to determine whether any was the result of a mutation affecting initiation of sporulation. Mutations to asporogeny (spo) may affect sporulation at any of a number of stages, and they can be ordered in a temporal sequence according to the morphological stage at which they lead to arrest (Esposito et al., 1970; Esposito \& Esposito, 1974; Esposito \& Klapholtz, 1981). However, none can be definitely assigned to initiation since there are no early events known which can be used as markers for initiation.

Asporogenous mutants defective in initiation can, however, be selected. It is possible to obtain populations of cells growing under conditions in which a proportion of the cells are sporulating. If the cells are dividing with a specific fission rate of $v$, and are being initiated to

Abbreviations: TTC, triphenyltetrazolium chloride; KAc, potassium acetate. 
sporulate at a specific rate $\kappa$, then the population of wild-type cells will have a reduced effective specific number growth rate (approximately $\nu-\kappa$ ). If a mutation occurs preventing the cells from initiating sporulation, the mutant will have an effective division rate of $v$, and will outgrow the wild-type cell, and also any later-blocked asporogenous mutants, since these will also have reduced growth rate. This can only succeed if there are some common elements regulating the decision by cells to initiate cell division on the one hand and sporulation on the other. Studies with derepressed mutants have indicated that there may indeed be common elements and that this approach is feasible, since these mutants are unable to grow on many non-fermentable substrates, but sporulate extensively instead (Vezinhet et al., 1979). These mutants cultured on such substrates frequently give rise to double mutants in which a second mutation to asporogeny suppresses the derepression on mutation and restores the ability of the cell to grow on nonfermentable substrates (Dawes \& Calvert, 1984). A second approach to isolating initiationdefective mutants relies on the behaviour of sporulating micro-organisms in continuous culture, and is analogous to the methods used to isolate the very early-blocked spoO mutants in Bacillus subtilis (Piggot \& Coote, 1976). Bacillus subtilis can be maintained in a chemostat such that some cells are sporulating while the rest maintain vegetative growth (Dawes \& Mandelstam, 1970), and under these conditions the population growth rate is less than the division rate of the vegetative cells, since cells initiated to sporulation cease to contribute to population growth (Dawes \& Thornley, 1970). In such populations, only asporogenous initiation mutants have a selective advantage, since all of their progeny can divide; any other (later-blocked) spo mutations would not confer this advantage. Saccharomyces cerevisiae can also undergo sporulation in continuous culture (Calvert \& Dawes, unpublished) and hence it should be possible to obtain spo initiation mutants from wild-type strains in this way.

This paper describes the isolation of putative spo initiation mutations using the methods outlined above, and the subsequent biochemical and genetic characterization of the mutations. These mutations confer a number of interesting properties that set them apart from other spo mutations.

\section{METHODS}

Organisms. The main strains used in addition to those given in Dawes \& Calvert (1984) included ST34 (MAT adc28-15 arol lys2 cyhl from S. Reed, Santa Barbara University of California, USA), BR207.7a (MATa cdc25-3 adel/ade2 ural trpl hisl arg4 ilvl) and BR220-169a (MAT\&cdc35-4 adel/ade2 ural trpl arg4) from J. Pringle (University of Michigan, Ann Arbor, USA). Strains carrying the whil and whi2 size control mutations (Carter \& Sudbery, 1980; Sudbery et al., 1980) were obtained from P. Sudbery (University of Sheffeld, UK).

Media. Complex media contained, $\left(1^{-1}\right): 10 \mathrm{~g}$ yeast extract, $20 \mathrm{~g}$ bacto-peptone and $20 \mathrm{~g}$ carbon source (YEPD contained glucose; YEPA, potassium acetate; YEPGal, galactose) except YEPG (30 $\mathrm{ml}$ glycerol) and YEPE $\left(20 \mathrm{ml}\right.$ ethanol). Minimal medium contained, $\left(1^{-1}\right): 1.5 \mathrm{~g}$ Difco yeast nitrogen base (without amino acids or ammonium sulphate), $5 \mathrm{~g}$ ammonium sulphate and $20 \mathrm{~g}$ glucose, and was supplemented with appropriate mixtures of amino acids to assign auxotrophic markers. Where needed, media were solidified with agar $\left(20 \mathrm{~g}^{-1}\right)$. Potassium acetate sporulation medium (KAc) was that of Fast (1970).

Contimuous cultivation. Strains were grown in a New Brunswick C-30 bench-top fermenter ( $305 \mathrm{ml}$ working volume). Temperature was maintained at $30^{\circ} \mathrm{C}$ using a cooling probe and a thermistor-controlled heating element. Agitation was maintained at 800 r.p.m. and the culture was aerated with sterile water-saturated air at 78 culture volumes $\mathrm{h}^{-1}$. Dilution rate was controlled by a peristaltic pump (Pharmacia) and samples were taken directly from the culture vessel using a hooded sampler, discarding the first sample, which contained material from the sampling line dead space. Cultures were grown under galactose limitation using a medium containing, (1-1): $1 \mathrm{~g}$ galactose (autoclaved separately); $1.5 \mathrm{~g}$ Difco yeast nitrogen base (without amino acids or ammonium sulphate); $2 \mathrm{~g}$ ammonium sulphate, $0.05 \mathrm{~g}$ L-arginine; $3 \mathrm{~g} \mathrm{KH}_{2} \mathrm{PO}_{4}$; and $5 \mathrm{~g} \mathrm{~K} \mathrm{HPO}_{4}$.

Tetrazolium reduction. By adapting the method of Ogur $e t$ al. (1957), strains were tested for their ability to reduce tetrazolium dye. Cultures grown on agar plates were overlaid with 15 ml molten agar $\left(50^{\circ} \mathrm{C}\right)$ containing $\left(1^{-1}\right): 0 \cdot 1 \mathrm{~g}$ triphenyltetrazolium chloride (TTC) and $20 \mathrm{~g}$ agar buffered at pH 7.2 with $0.1 \mathrm{M}$-potassium phosphate. Clones of tetrazolium-reducing cells became red within 2 to $3 \mathrm{~h}$.

Genetic analysis. Standard techniques of genetic analysis were used (Mortimer \& Hawthorne, 1975). Crosses involving homothallic diploid strains were made as described by Dawes \& Calvert (1984). 
RESULTS

\section{Isolation of 'revertants' from a homozygous spd1 diploid}

Once mutations affecting a process such as initiation of sporulation are available, further mutations affecting the process can be obtained by selecting for 'reversion' of the original mutant phenotype and screening for the occurrence of second-site mutations suppressing the initial mutation. For spd mutations leading to derepressed sporulation this approach is facilitated by their instability: homozygous $s p d l$ diploids plated on media on which they cannot grow, but on which they sporulate extensively, spontaneously give rise to colonies at a frequency of around $4 \times 10^{-4}$ (Dawes \& Calvert, 1984).

Lawns of the homothallic diploid strain 54 (spdl homozygote) grown on YEPD plates after replica-plating onto YEPG plates gave numerous papillae after incubation for two weeks. From a genetic analysis of 34 independently isolated clones it was found that more than half of these retained the spdl mutation (Table 1) and the revertant phenotype was the result of the acquisition of a second, unlinked mutation. In 10 of the 20 such cases the second-site mutation was present in heterozygous state, and these strains grew on YEPG and sporulated on sporulation plates. On dissection of asci from these, diploids homozygous for the mutation suppressing the inability of spdl strains to grow on YEPG were obtained. These were found to be asporogenous under all conditions, and the mutation suppressing $s p d l$ was therefore acting as a recessive spo mutation.

Ten of the 'revertants' were asporogenous on isolation and were found to have resulted from the acquisition of an spo mutation in the homozygous state. These homozygous diploids were difficult to analyse by meiotic analysis, since back-crossing to wild-type strains could only be achieved in some of them, and this involved incubating them for an extended time on sporulation medium before attempting to mate them to a wild-type haploid. Under these conditions a very few spores formed in the spo strains and these could be induced to mate to give diploids heterozygous at the $H O$ (homothallism), spdl and spo loci. These were sporulated and dissected to isolate the spo mutation in an SPDI background. For three independent spo mutations tested in this way, complementation tests were performed by isolating MATa and MAT $\alpha$ haploids carrying the spo mutation. All three mutations from revertant strains R42, R65 and R77 failed to complement each other and were presumed to represent different alleles of the same locus. From the dissections used to characterize R42, strain $69.10 \mathrm{C}$, a homothallic diploid homozygous for the spo50 mutation, was obtained and used in subsequent analysis.

\section{Isolation of 'revertants' from a haploid spdl strain}

Isolation of the spo mutations in a homothallic diploid strain led to considerable difficulty in genetic analysis. This problem was largely avoided by isolating 'revertants' from a haploid strain, 168.2D (MATa spd1-1 metl) grown on YEPG medium. These 'revertant' strains were back-crossed to a wild-type strain, 168.2A (MATa arg4-1), and the resultant cross dissected. Clones with genotypes MATa spo arg4-1 and MAT $\alpha$ spo metl were obtained and mated, and each resultant cross was tested for its ability to sporulate. From 20 isolates four classes were obtained; these included: three mating-deficient strains which could not be analysed further; three wild-type revertants producing four normally-sporulating clones from each ascus; six unstable 'revertants' which after mating and dissection had recovered the spd1-1 phenotype; and six stable 'revertants'. On backcrossing to the wild-type strain, the spdl-1 mutation was recoverable from these latter isolates, indicating that the mutation restoring growth on YEPG was at a second site unlinked to the spd1-1 locus. When these second-site suppressor mutations were present in the homozygous state, they conferred asporogeny.

\section{Isolation of an spo mutant from continuous culture}

Strain $\mathrm{S} 41$ was grown in a galactose-limited chemostat culture, as described in Methods. The culture reached a peak of sporulation of $35 \%$ asci, which was maintained for approximately $100 \mathrm{~h}$, after which asporogenous mutants appeared in the culture and these very rapidly became the predominant organisms. These mutants were obtained in pure culture and proved to have very similar characteristics to the other asporogenous mutants described above. 
Table 1. Analysis of spontaneous 'revertants' of a homozygous spdl diploid strain

Strain 54 was plated on YEPG plates at high cell density. After incubation for two weeks papillae were picked, purified by streaking on YEPG plates and tested for their ability to sporulate on plates of sporulation medium. Asci from those isolates that sporulated to any extent were dissected and the progeny scored for derepressed sporulation, normal sporulation or absence of sporulation on appropriate media. Three classes of mutant were obtained.

\begin{tabular}{|c|c|c|c|c|c|}
\hline \multirow{2}{*}{$\begin{array}{l}\text { No. of } \\
\text { mutants } \\
\text { isolated }\end{array}$} & \multirow{2}{*}{$\begin{array}{l}\text { Percentage } \\
\text { sporulation }\end{array}$} & \multicolumn{3}{|c|}{ Nos of tetrads } & \multirow{2}{*}{$\begin{array}{l}\text { Presumed } \\
\text { genotype }\end{array}$} \\
\hline & & Wild-type & Derepressed & Asporogenous & \\
\hline 14 & $70-90$ & 4 & 0 & 0 & $\frac{S P D I}{S P D I}$ \\
\hline 10 & $10-50$ & 0 & 2 & $2 *$ & $\frac{s p d 1-1}{s p d l-1} \frac{s p o}{S P O}$ \\
\hline 10 & $\mathbf{0}$ & - & - & - & $\frac{s p d l-1}{s p d l-1} \frac{s p o}{s p o}$ \\
\hline
\end{tabular}

* These asporogenous strains had highly pleiotropic phenotypes similar to those observed for the totally asporogenous mutants.

Table 2. Growth and sporulation phenotypes of diploids carrying different combinations of spd1 and spo50 mutations

Strains with the genotypes listed below were constructed from the parent strains: $\$ 41$ (wild type), 54 (spdI) or 69.10C (spo50), or from the progeny of appropriate crosses between these strains. At least 5 isolates with each genotype were obtained, and the extent of sporulation was assessed on YEPG and KAc media. The figures indicate the range of sporulation frequencies observed.

\begin{tabular}{|c|c|c|c|c|}
\hline \multirow{2}{*}{$\begin{array}{c}\text { Strain } \\
\text { genotype }\end{array}$} & \multicolumn{2}{|c|}{ Growth } & \multicolumn{2}{|c|}{ Percentage sporulation (48h) } \\
\hline & YEPD & YEPG & YEPG & KAC \\
\hline$\frac{S P D 1}{S P D 1} \frac{S P O S 0}{S P O S 0}$ & + & + & $0-5$ & $60-80$ \\
\hline$\frac{\text { SPdI }}{S P D 1} \frac{S P O S O}{S P O S O}$ & + & + & $5-10$ & $60-80$ \\
\hline$\frac{\text { spdl }}{\text { spdl }} \frac{S P O S O}{S P O S O}$ & + & - & $70-95$ & $70-95$ \\
\hline$\frac{S P D I}{S P D D} \frac{\text { sposo }}{S P O S 0}$ & + & + & $0-5$ & $50-80$ \\
\hline$\frac{S P D 1}{\text { SPD1 }} \frac{\text { sposo }}{\text { Spos0 }}$ & + & + & 0 & $0-5$ \\
\hline$\frac{\text { spdl }}{\text { spdl }} \frac{\text { sposo }}{\text { spo } 50}$ & + & + & $\mathbf{0}$ & $0-10$ \\
\hline
\end{tabular}

\section{Dominance and complementation analysis}

Diploids heterozygous for the above asporogenous mutations were constructed and tested for their ability to sporulate on sporulation medium. The mutations included those in the six haploid asporogenous isolates, the chemostat-isolated strain $\mathrm{CH} 15$, and the diploid 69.10C. All of these were recessive to the wild-type with respect to their effect on sporulation, and therefore complementation tests were done. It should be noted, however, that these mutations were dominant with regard to suppression of the spdl characteristic of poor growth on nonfementable carbon sources. The growth and sporulation characteristics of diploids carrying the various combinations of wild-type and mutant alleles of spdl and sposo are summarized in Table 2.

Three complementation groups for these potential initiation spo mutations were established (Table 3). Crosses between strains carrying mutations from different groups all yielded diploids sporulating at a frequency over $50 \%$, while those between strains carrying mutations in the same complementation group were asporogenous. 
Table 3. Complementation analysis of mutations to asporogeny that suppress the spdl mutation

The numbers refer to strains in which independent mutations were first characterized. For haploid strains, opposite mating types were constructed from dissection of a cross to a wild-type haploid. For diploids $69.10 \mathrm{C}$ and $\mathrm{CH} 15$ the appropriate heterozygous diploids were constructed by crossing the very few spore progeny of each strain to the haploids. Heterozygous diploids were tested for sporulation on KAc sporulation plates; a plus sign indicates that complementation had occurred and sporulation (> 50\%) was obtained.

\begin{tabular}{|c|c|c|c|c|c|c|c|c|c|c|c|}
\hline $\begin{array}{l}\text { Parent } \\
\text { strain }\end{array}$ & $\begin{array}{l}\text { Genotype } \\
\text { designated }\end{array}$ & & & & & & & & & & \\
\hline $69.10 \mathrm{C}$ & spo50-1 & - & & & & & & & & & \\
\hline $\mathrm{r} 42$ & spo50-2 & - & - & & & & & & & & \\
\hline r77 & spo50-3 & - & - & - & & & & & & & \\
\hline 2 & spo51-1 & + & $*$ & $*$ & - & & & & & & \\
\hline 8 & spo51-2 & + & $*$ & * & - & - & & & & & \\
\hline 4 & spo53-1 & + & * & $*$ & + & + & - & & & & \\
\hline 6 & spo53-2 & + & $*$ & $*$ & + & + & - & - & & & \\
\hline 10 & spo53-3 & + & $*$ & $*$ & + & + & - & - & - & & \\
\hline 14 & spo53-4 & + & $*$ & * & + & + & - & - & - & - & \\
\hline \multirow[t]{2}{*}{$\mathrm{CH} 15$} & spo53-5 & + & $*$ & $*$ & $*$ & + & $*$ & $*$ & - & $*$ & - \\
\hline & & $69.10 \mathrm{C}$ & $\mathrm{r} 42$ & r77 & 2 & 8 & 4 & 6 & 10 & 14 & $\mathrm{CH} 15$ \\
\hline
\end{tabular}

\section{Linkage analysis for sporulation initiation mutations}

From the heterozygous diploids constructed for the above complementation tests, representative strains were dissected to determine the linkage relationships between spo50, spo51 and spo53. Additionally, the other mutations affecting sporulation initiation, including: $s p d 1, s p d 3, s p d 4$ (derepressed sporulation mutations; Dawes \& Calvert, 1984), cdc25, cdc28, cdc35 (temperature-sensitive cell division cycle mutations; Hartwell, 1974; Reed, 1980; Shilo et al., 1978), whil and whi2 (mutations affecting cell size; Sudbery et al., 1980) were tested for linkage by meiotic analysis using tetrad dissection where this was feasible, or random spore analysis when lack of spore viability made tetrad analysis unfeasible. No close linkage of any of the above mutations was detected, although some linkage of $s p d l$ and spo53 was inferred from the low frequency of non-parental ditypes in dissection (Table 4). From the data obtained, it can be calculated that spo53 is about $35 \mathrm{cM}$ from spd1. The spd1 mutation is centromere-linked (Dawes $\&$ Calvert, 1984) and from this fact and the data in Table 4, spo50, spd3 and spo53 are also linked to their respective centromeres. The probable location of spo53 is the right arm of chromosome IV, about $20 \mathrm{cM}$ from the centromere, although poor spore viability has prevented a more definite assignment by tetrad analysis.

In the course of linkage tests, it was also shown that strains carrying any of the three spo mutations and $s p d 3$ failed to express the spd phenotype. Thus all three suppressors (spo50, spo51 and spo53) of spdl were also able to suppress spd3, implying that all of these mutations affect a common function in the cell.

\section{The spo50, spo51 and spo53 mutations are not nonsense suppressors}

The high frequency with which spo50, spo5 1 and spo53 arose in strains carrying spdl, and their ability to suppress spd3 mutations, may have been the result of the spd mutations being nonsense suppressible alleles, and the spo mutations being amber, ochre or opal suppressors. This was ruled out in two ways. Diploids were constructed which were homozygous for spdl and various combinations of known amber, ochre and opal mutations, including: arg4-17, his5-2, lys 1-2 (ochre); trp1-1, aro7-1 (amber); ade5,7-u; his5'-u; leu2'-u; lys1-u (opal; Hawthorne, 1976). On isolating at least 20 spontaneously arising asporogenous clones that grew on YEPG, none was found to have lost any of the auxotrophic requirements, showing that the mutations to asporogeny failed to suppress the nonsense-suppressible markers. In the same way nonsense suppressors selected by simultaneous suppression of several auxotrophic markers were found 
Table 4. Meiotic analysis of mutations affecting initiation of sporulation

Diploids heterozygous for the various combinations of the mutations indicated were sporulated and dissected. Where feasible, tetrads were scored for the frequencies of parental ditype (PD), nonparental ditype (NPD) and tetratype (T) segregation. For the $c d c$ mutations poor spore viability was a problem and random spore analysis was used instead.

\begin{tabular}{|c|c|c|c|c|c|}
\hline & \multirow{2}{*}{\multicolumn{3}{|c|}{ Tetrad analysis }} & \multicolumn{2}{|c|}{ Random spore analysis } \\
\hline & & & & \multirow{2}{*}{$\begin{array}{l}\text { No. of } \\
\text { spores }\end{array}$} & \multirow{2}{*}{$\begin{array}{c}\text { Spores of } \\
\text { parental type }(\%)\end{array}$} \\
\hline & PD & NPD & $\mathbf{T}$ & & \\
\hline$s p d 1 \times s p o 50$ & 4 & 5 & 10 & 93 & $(54)^{*}$ \\
\hline spd I $\times$ spo5I & 1 & 1 & 7 & 75 & $(64)^{*}$ \\
\hline spdl $\times$ spos3 & 14 & 1 & 15 & 68 & $(70)^{*}$ \\
\hline spd3 $\times$ sposo & 10 & 10 & 15 & 117 & $(62)^{*}$ \\
\hline spd3 $\times$ spos 51 & 7 & 5 & 6 & 89 & $(54)^{*}$ \\
\hline$s p d 3 \times s p o 53$ & 1 & 0 & 5 & 56 & $(50)^{*}$ \\
\hline spo50 5 spo5 51 & 9 & 3 & 11 & 134 & $(46)^{*}$ \\
\hline sposo 5 spos3 & 4 & 6 & 6 & 89 & $(62)^{*}$ \\
\hline spos $51 \times s p 053$ & 4 & 5 & 20 & 129 & $(54)^{*}$ \\
\hline spdl $\times$ whil & 3 & $\mathbf{I}$ & 6 & 70 & 58 \\
\hline spd $1 \times$ whi2 & 6 & 2 & 17 & 100 & 58 \\
\hline sposo $\times$ whil & 2 & 1 & 5 & 32 & 53 \\
\hline spo50 $\times$ whi2 & 1 & 4 & 5 & 40 & 43 \\
\hline sposI $\times$ whi2 & 2 & 1 & 4 & 44 & 64 \\
\hline spos $3 \times$ whil & 3 & 6 & 7 & 88 & 47 \\
\hline spo53 3 whi2 & 2 & 4 & 5 & 36 & 23 \\
\hline$s p d I \times c d c 25$ & & & & 39 & 24 \\
\hline spdl $\times$ cdc35 & & & & 35 & 34 \\
\hline sposo $\times$ cdc 28 & & & & 17 & 47 \\
\hline spo $51 \times c d c 25$ & & & & 16 & 68 \\
\hline spos $1 \times c d c 28$ & & & & 29 & 48 \\
\hline spos $1 \times c d c 35$ & & & & 6 & 33 \\
\hline $50053 \times c d c 25$ & & & & 11 & 45 \\
\hline spo53 $\times$ cdc 28 & & & & 16 & 44 \\
\hline spo53 0 cdc35 & & & & 13 & 61 \\
\hline
\end{tabular}

- In these crosses only one class of parental spore type could be easily distinguished (spd), and the percentage given is double that for this class.

neither to suppress the YEPG growth phenotype of the spdl mutation nor to produce asporogeny. Some strains isolated sporulated poorly, but this can be accounted for by the known characteristics of some nonsense suppressor mutations (Rothstein et al., 1976). In addition, the ochre-specific nonsense suppression mutation SUP3, and opal suppressors SUP76 and SUP85 (Hawthorne, 1976) were found not to suppress the spdl mutation. These results show that the second-site mutations to asporogeny are not nonsense suppressors, nor were any of the four alleles of the spdl mutation tested nonsense suppressible.

\section{Pleiotropic phenotypes of spo50, spo51 and spo53 mutations}

Diploids homozygous for any of these three asporogenous initiation mutations exhibited similar highly pleiotropic phenotypes that set these mutations apart from others causing asporogeny.

Starvation response. One characteristic noticed in the routine handling of spo50 and spo51 mutants was their extensive loss of viability on prolonged incubation on plates and in storage on agar slopes kept at $4^{\circ} \mathrm{C}$. Fig. 1 illustrates the marked decrease in survival of these strains compared to the wild-type in cultures grown to maximum turbidity in YEPA medium. For the homozygous spo50 diploid there were fewer than $0.1 \%$ survivors after $5 \mathrm{~d}$ further incubation compared with about $60 \%$ for the wild-type. Under these conditions the asporogenous mutants failed to adopt a normal stationary-phase morphology characterized by single unbudded cells (appearing phase-bright); instead they became aberrant, producing long processes without completing cytokinesis (Fig. 2). This aberrant morphology occurring under starvation 


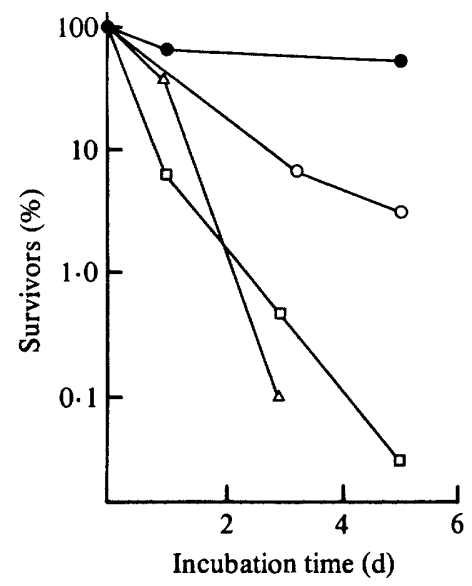

Fig. 1. Viability of mutant diploid strains on prolonged incubation. Strains were grown to stationary phase at $30^{\circ} \mathrm{C}$ with shaking in YEPA medium, then monitored for change in viable cell numbers on continued incubation. Homothallic diploid wild-type strain S41; 0 , homozygous spo50 diploid strain 69.10C; $\triangle$, homozygous spo51 diploid strain 246.1B; $\square$, homozygous spo53 diploid strain 247.2D.

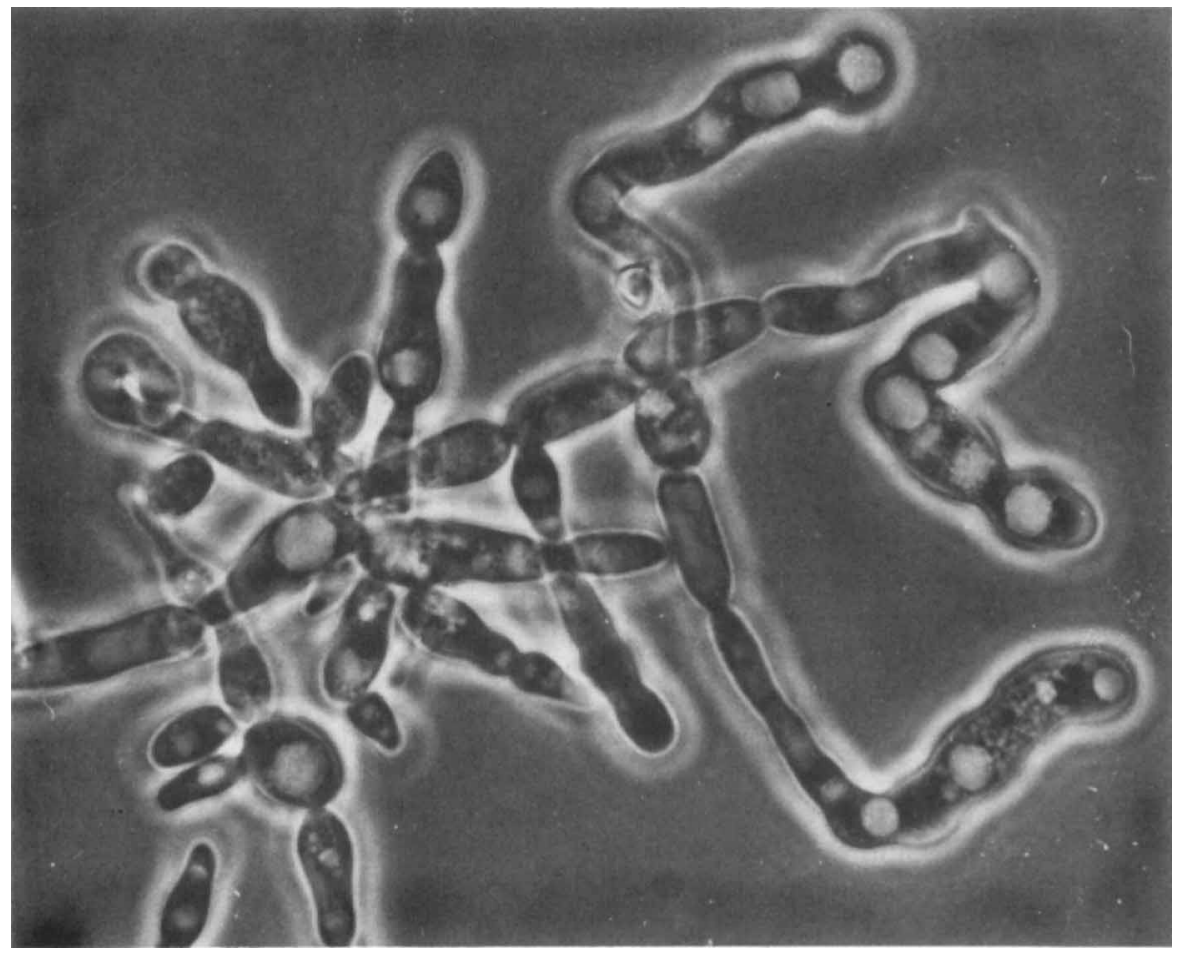

Fig. 2. Photomicrograph of homozygous spo50 diploid strain $69.10 \mathrm{C}$ grown to stationary phase. The pseudomycelial appearance of this strain does not begin to develop until stationary phase has been attained (the above was obtained from a culture incubated for $48 \mathrm{~h}$ in YEPA medium at $30^{\circ} \mathrm{C}$ ). Magnification approx. $1700 \times$.

conditions was characteristic of spo50, spo51 or spo53 strains and may indicate that the cells were continuing cell growth, but that the cytokinesis and cell separation events of the cell division cycle were prevented.

The colonies of homozygous spo50, spo51 or spo53 diploids could easily be distinguished from 
those of wild-type strains since they became a distinct brown colour after prolonged incubation on YEPD agar. The surface of these colonies often became crinkled and cells in the colonies were often tightly bound so that they were difficult to replicate. This was probably due to the pseudomycelial morphology of the strains seen after starvation.

Reduction of TTC. The spdl mutation may affect some aspect of mitochondrial activity (Vezinhet et al., 1979) and in view of the ability of the spo50, 51 and 53 mutations to suppress the spdl mutation they may also affect mitochondrial activity. Several strains were therefore tested for their ability to reduce TTC via the respiratory chain (Slater et al., 1963), and distinct differences were observed between spdl, spo50, spo51, spo53 and wild-type strains. The wildtype strain did not reduce TTC when fermenting glucose, but reduced it strongly when growing by respiration of glycerol. The spdl strain 54, however, failed to reduce TTC on any medium tested and was apparently unable to respire. By contrast, strains carrying any of the above spo mutations were able to reduce TTC, even when grown on glucose. The characteristic was sufficiently reliable for its use as a diagnostic test in screening for spo initiation mutations, or scoring for their presence, and indicated that in such strains respiratory functions are constitutively derepressed.

Differences in protein composition. Several differences between the protein compositions of wild-type and spdI strains have been detected by polyacrylamide gel electrophoresis. One polypeptide of molecular weight about 21000 whose appearance is partially derepressed in spdl strains was not produced by spo50 mutants under any conditions of growth including those (on YEPG or YEPE) in which the wild-type strains do produce this polypeptide (Dawes \& Calvert, 1984).

\section{DISCUSSION}

A further three genes, SPOSO, SPOSI and SPOS3, have been implicated in the initiation of meiosis and sporulation, in addition to those previously reported, including $C D C 25, C D C 35$, possibly CDC28 (Shilo et al., 1978), SPD1, SPD3 and SPD4 (Dawes \& Calvert, 1984). Mutation in any of these three $S P O$ genes invariably resulted in cells trying to divide under conditions that normally favour sporulation. They act as by 'bypass' mutations suppressing derepressed sporulation in an spdl or spd3 background and confer a growth advantage under conditions in which a proportion of a wild-type population would sporulate. Diploids homozygous for these spo mutations showed none of the morphological changes associated with sporulating cells and, moreover, a strain homozygous for the spo50 mutation did not undergo premeiotic DNA replication or meiotic recombination under normal sporulation conditions, although a few very early sporulation-specific polypeptide changes have been detected in this strain (Ajam, 1981; Wright et al., 1981). These changes occurred in wild-type cells well before they became committed to meiosis.

While the spo50, spo51 and spto53 mutations promoted cell division at the expense of sporulation, diploids homozygous for them were apparently unable to adopt a normal stationaryphase configuration under starvation conditions. Wild-type cells in stationary phase fail to initiate sporulation (Hirschberg \& Simchen, 1977) and assume distinctive shapes, chromosome structure (Piñon, 1978) and staining reactions (Schwartzenruber, 1977). Piñon suggested that stationary phase is not simply arrest in Gl of the cell cycle, but is a separate life cycle phase (designated G0) with specific changes adapting the cell to starvation. Bedard et al. (1981) doubted the existence of a separate $\mathbf{G 0}$ phase as they were unable to isolate mutants that were specific to it; our isolates may be such mutants.

The spo50, spo51 and spo53 genes therefore have a role in determining whether the cell initiates sporulation, continues division or adapts to starvation. They act in a positive way to promote the initiation of sporulation and adaptation to starvation conditions, as mutations in them leading to asporogeny and abnormal starvation morphology are recessive. However, the same mutations are at least partially dominant in suppressing the inability of $s p d l$ strains to grow on non-fermentable carbon sources.

Since mutation at any of the spo50, spo5I or spo53 loci suppresses any of the spdl or spd3 
mutations, and since they restore to $s p d$ strains the ability to grow on glycerol, it is likely that all five mutations affect a common function in the cell. The spd1 mutations appear to influence directly the mechanism by which cells monitor their nutritional status when respiring, and this may involve some mitochondrial function (Dawes \& Calvert, 1984). The loss of glucose repression of TTC reduction in the spo50, spo51 and spo53 mutants supports this hypothesis and the mutants that have lost glucose control over mitochondrial activity recently described by Böker-Schmitt et al. (1982) should be considered in this context since they have a similar phenotype.

In summary, it seems likely that the decision between continued cell division or initiation of meiosis and spore formation depends on the level of some mitochondrial activity encoded by the nuclear $S P D$ genes. A reduction in this activity by mutation could be compensated for by derepression of mitochondria under the control of the $S P O$ genes described here. Sporulation is known to be dependent on respiration (Roth \& Halvorson, 1969) or at least on some mitchondrial functions (Pratje et al., 1979; Hartig \& Breitenbach, 1980), yet mutations that lead to one form of respiratory deficiency (spd1, spd3 and $s p d 4)$ promote sporulation, while those probably resulting in derepressed mitochondrial activity (spo50, spo51 and spo53) inhibit sporulation. There is therefore a more fundamental and complex regulatory connection between respiration and sporulation than the simple dependence of sporulation on respiration. Definition of this connection requires a detailed analysis of mitochondrial metabolism as cells are shifted from growth to sporulation conditions, using wild-type strains and the mutants described here.

This work was supported by grants from the SERC and an SERC studentship award to G. R. C.

\section{REFERENCES}

AJAM, N. (1981). Regulation of sporulation in Saccharomyces cerevisiae, pp. 151-174. PhD thesis, University of Edinburgh, UK.

Bedard, D. P., Johnston, G. C. \& Singer, R. A. (1981). New mutations in the yeast Saccharomyces cerevisiae affecting completion of 'start'. Current Genetics 4, 205-214.

BökER-SChMitT, E., FranCISCI, J. \& SCHWEYEN, R. S. (1982). Mutations releasing mitochondrial biogenesis from glucose repression in Saccharomyces cerevisiae. Journal of Bacteriology 151, 303-310.

CARTer, B. L. A. \& Sudbery, P. (1980). Small-sized mutants of Saccharomyces cerevisiae. Genetics 96, 561-566.

DAWES, I. W. (1975). Study of cell development using derepressed mutations. Nature, London 255, 707708.

Dawes, I. W. \& CalverT, G. R. (1984). Initiation of sporulation in Saccharomyces cerevisiae. Mutations causing derepressed sporulation and G1 arrest in the cell division cycle. Journal of General Microbiology 130, 605-613.

Dawes, I. W. \& Thornley, J. H. M. (1970). Sporulation in Bacillus subtilis. Theoretical and experimental studies in continuous culture systems. Journal of General Microbiology 62, 49-66.

Esposito, M. S. \& Esposito, R. E. (1969). The genetic control of sporulation in Saccharomyces. I. The isolation of temperature-sensitive sporulation-deficient mutants. Genetics 61, 78-89.

Esposito, M. S. \& Esposito, R. E. (1974). Genes controlling meiosis and spore formation in yeast. Genetics 78, 215-225.

Esposito, M. S., Esposito, R. E., ARnaud, M. \&
Halvorson, H. O. (1970). Conditional mutants of meosis in yeast. Journal of Bacteriology 104, 202-210.

EsPosito, R. E. \& KLAPHOLz, S. (1981). Meiosis and ascospore development. In The Molecular Biology of the Yeast Saccharomyces: Life Cycle and Inheritance, pp. 211-287. Edited by J. N. Strathern, E. W. Jones \& J. R. Broach. New York: Cold Spring Harbor Laboratory.

FAST, D. (1973). Sporulation synchrony in yeast. Journal of Bacteriology 116, 925-930.

Hartig, A. \& Breitenbach, M. (1980). Sporulation in mitochondrial oxi3 mutants of Saccharomyces cerevisiae. A correlation with the genetic map. Current Genetics 1, 97-102.

HARTWELL, L. H. (1974). Saccharomyces cerevisiae cell cycle. Bacteriological Reviews 38, 164-198.

HAWTHORNE, D. C. (1976). UGA mutations and UGA suppressors in yeast. Biochimie 58, 179-182.

HiRsChberG, J. \& SimCheN, G. (1977). Commitment to the mitotic cell cycle in yeast in relation to meiosis. Experimental Cell Research 105, 245-252.

MORTIMER, R. \& HAWTHORNE, D. C. (1975). Genetic mapping in yeast. Methods in Cell Biology 11, 221234.

OguR, M., St. John, R. \& Nagal, S. (1975). Tetrazolium overlay technique for population studies of respiration deficiency in yeast. Science 125, 928-929.

Piggot, P. J. \& CoOTE, J. G. (1976). Genetic aspects of bacterial endospore formation. Bacteriological Reviews 40, 908-962.

PIÑON, R. (1978). Folded chromosomes in non-cycling yeast cells. Evidence for a characteristic G0 form. Chromosoma 67, 263-274. 
Pratje, E., Schulz, R., SchNierer, S. \& Michablis, G. (1979). Sporulation of mitochondrial respiratorydeficient mit- mutants of Sacchanomyces cerevisiae. Molecular and General Genetics 176, 411-415.

REED, S. I. (1980). The selection of Saccharomyces cerevisiae mutants defective in the start event of cell division. Genetics 95, 561-577.

ROTH, R. \& Halvorson, H. O. (1969). Sporulation of yeast harvested during logarithmic growth. Joumal of Bacteriology 98, 831-832.

Rothstein, R. J., Espostro, R. E. \& Esposito, M. S. (1976). The effect of ochre suppression on meiosis and ascospore formation in Saccharomyces. Genetics 85, 35-54.

Shlo, V., Smachen, G. \& Shlo, B. (1978). Initiation of meiosis in cell-cycle initiation mutants of Saccharomyces cerevisiae. Experimental Cell Research 112, 241-248.

Slater, T. F., Sawyer, B. \& Sträul, U. (1963). Studies on succinate-tetrazolium reductase system.
11I. Points of coupling of four different tetrazolium salts. Biochimica et biophysica acta 77, 383-393.

Sudbery, P. E., GoOdey, A. R. \& Carter, B. L. A. (1981). Genes which control cell proliferation in the yeast Saccharomyces cerevisiae. Nature, London 288 , 401-404.

SWARTZENDRUBER, D. E. (1977). A bromodeoxyuridine (BUdR)-mithramycin technique for detecting cycling and non-cycling cells by flow microfluorimetry. Experimental Cell Research 109, 439443.

VEZINHET, F., KINNAIRD, J. H. \& DAWES, I. W. (1979). The physiology of mutants derepressed for sporulation in Saccharomyces cerevisiae. Journal of General Microbiology 115, 391-402.

Wright, J. F., AJAM, N. \& DAWES, I. W. (1981). The nature and timing of sporulation-specific protein changes in Saccharomyces cerevisiae. Molecular and Cellular Biology 1, 910-918. 